\title{
Análisis y perspectivas de la profesionalización de la Administración Pública en México
}

Rafael M artínez Puón*

\section{Introducción}

En el marco de la reforma de los aparatos gubernamentales y administrativos, una de las actividades más importantes por lograr, sin duda alguna, es la relacionada con la profesionalización del personal que forma parte de las Administraciones Públicas. Las propuestas en este terreno son diversas, desde aque llas que tienen que ver con las nuevas tecnologías administrativas como la G estión Estratégica de Recursos H umanos (Strategic H uman Resources M anagment) (AM STRONG, 1990; KLIG NER y N ALBADIAN 1998) que forma parte de todo el movimiento denominado Nueva Gestión Pública, hasta aquellas que se enmarcan en la posibilidad de establecer elementos del modelo burocrático weberiano, específicamente lo que es la figura de un servicio civil de carreral sobre todo en países en los que no existe, al gunos países de Latinoamérica y particularmente el caso de M éxico.

Desde hace algunos años, el Estado mexicano, al igual que los demás países de Latinoamérica, ha experimentado una serie de reformas principalmente de carácter económico y de carácter político, pero también ha comenzado a realizar reformas Ilamadas de tercera generación, por orden de importancia, que tienen que ver principalmente con la reforma en la gestión pública y la modernización administrativa² (M ÉNDEZ, 1998a: 26). Por lo que el cambio tiende a ser integral y no parcial.
Los avances en reformas de tercera generación tanto en actividades y procesos han sido de todo tipo: desde la prestación de servicios públicos, nuevas relaciones con los ciudadanos, la adopción de nuevas tecnologías, descentralización administrativa y financiera hasta la propia profesionalización del personal de las Administraciones Públicas. Sin embargo, todavía prevalecen algunas metas por alcanzar y el tema de la profesionalización de servidores públicos no es la excepción, sobre todo cuando la realidad de la gran mayoría de los servidores públicos en M éxico en sus tres niveles de gobierno sigue marcada por una incertidumbre laboral generada por el propio sistema jurídico y político imperante, que es propicio a la designación política o sistema de patronazgo (Prats, 1998) o como se conoce en los países anglosajones como spoil system, que en más de las veces privilegia la confianza y no los méritos profesionales de las personas. Los incesantes cambios en los servidores públicos que se registran periódicamente (sobre todo aquellos que forman parte de la franja en la que se toma en el mayor número de decisiones) no han permitido el establecimiento de un sistema permanente de profesionalización ni todo un sistema de carrera administrativa.

En este tenor, se han experimentando una serie de modalidades diversas, - por cierto no muy recientes- bifurcadas en los enfoques antes mencionados (Gestión Estratégica de Re cursos H umanos/Weberianismo), éstas van desde la aplicación de sistemas de capacitación en las funciones que por ley presta toda institución pública; sistemas de capacitación alrededor de esquemas de calidad total (M artínez Puón, 2000: 40-41); 
profesionalización sobre la filosofía de la gerencia pública (Uvalle, 2000: 172); profesionalización en organismos sectoriales ${ }^{3}$, hasta el intento desde principios de los años ochenta de establecer un servicio civil de carrera con la creación de una Comisión Intersecretarial de Servicio Civil, y de manera reciente - sobre todo en los últimos 5 años- con la aparición de un número significativo de iniciativas de ley para la implementación de esta figura. Esfuerzos que no han sido lo suficientemente consistentes en su concreción para devolverle la legitimidad y la eficacia necesaria al gobierno y a la Administración Pública mexicana. Recientemente, a nivel de los gobiernos de los Estados de la República, un avance importante es el obtenido en el Distrito Federal que ya cuenta con una ley en la materia aprobada por su Asamblea Legislativa en junio del 2000 que ha comenzado a implementarse.

Esto ha dado lugar en el país, básicamente, a la concurrencia de dos visiones en materia de profesionalización de servidores públicos, la que se pronuncia por el movimiento de la Nueva G estión Pública y la segunda, por el establecimiento de un servicio civil de carrera.

Por lo tanto, se asiste a la observación de un dilema en la Administración Pública mexicana, que no es exclusivo de este país, que consiste, por una parte, en crear y consolidar líneas administrativas propias del modelo burocrático weberiano, en este caso el establecimiento de un servicio civil de carrera, y, por la otra, avanzar hacia las nuevas tecnologías administrativas de la N ueva G estión Pública. Sobre lo último, se han registrado una serie de innovaciones y adaptaciones en la Administración Pública mexicana en los últimos 15 años en el marco de la modernización administrativa y particularmente a través de los distintos programas de modernización administrativa y del más reciente que es el Programa $\mathrm{N}$ acional de Combate a la Corrupción y Fomento a la Transparencia y el D esarrollo Administrativo 2001-2006 y el M odelo Estratégico para la Innovación Gubernamental, además de los programas de modernización a nivel estatal y sectorial; y sobre el establecimiento de un servicio civil de carrera, es importante mencionar, que la Administración Pública mexicana - a pesar de los pocos avances- pretende establecer un servicio civil de carrera con un retraso de más de un siglo con respecto a países como Inglaterra, Francia, Estados Unidos (PARD 0, 1995: 277 y ss.), Alemania, y con un retraso de décadas con respecto a páses de América Latina como Argentina, Brasil, Costa Rica, Honduras, El Salvador y República Dominicana.

Actualmente, los países mencionados se encuentran en una etapa de franca revisión y renovación de sus propios servicios civiles de carrera ${ }^{4}$, y en algunos casos como los latinoamericanos la situación es de rediseño, extensión o flexibilización (MÉN DEZ, 1998B: 3; LONGO, 2000). Por lo que esas experiencias recientes también deberán tomarse en cuenta, para casos como el de M éxico que por primera vez pretende establecer una figura de estas características; en el que si bien ya existen esfuerzos importantes a nivel sectorial, prevalece el interés por adoptar esta figura para cubrir todo el espectro de la Administración Pública.

¿Cuál es la coyuntura actual en materia de profesionalización de servidores públicos? Sin lugar a dudas, el elemento que ha sido fundamental para que la actividad de la profesionalización en los órganos gubernamentales y administrativos haya cobrado una mayor importancia, se debe al factor democrático imperante en M éxico en los últimos quince años, materializado en elecciones transparentes, alternancia política no sólo ahora a nivel de los Estados de la República, sino recientemente en el seno de la propia figura presidencial y por el nuevo equilibrio en los poderes públicos generado por el juego plural que se está dando a nivel de fuerzas políticas; los casos más significativos son aquéllos en los que los poderes ejecutivos no tienen la mayoría en sus respectivos congresos y el caso más claro ahora mismo, es a nivel federal en el que la mayoría en el Congreso de la Unión tanto la Cámara de Diputados como la Cámara de Senadores pertenecen a un partido distinto al que ocupa la titularidad del Poder Ejecutivo Federal. D e igual forma, el propio factor democrático ha dado lugar también al fortalecimiento de la descentralización política y administrativa la cual se ha materializado en nuevas atribuciones y recursos para los Estados de la República, incluyendo ahora al D istrito Fede ral que recientemente acaba de adquirir la figura de entidad federativa con un Jefe de Gobierno electo por la ciudadanía. Todo ello, aunado a la presencia cada vez mayor de organismos civiles y de la ciudadanía en sí - como producto del propio escenario democrático - que demandan día a día mejores soluciones a los diferentes problemas que existen en el país, han ve nido a constituirse en su conjunto en los impulsores de un cambio en la Administración Pública y en la profesionalización de sus servidores públicos.

Por lo tanto, de acuerdo con las condiciones descritas, se vuelve imprescindible el hecho de contar con aparatos administrativos eficientes y profesionalizados tanto a nivel federal como estatal, con finanzas públicas sanas y con un manejo transparente de los recursos públicos; con un sentido de ética en el servicio público; con decisiones lo más democráticas posibles y con una prestación de servicios públicos de calidad y que brinde la seguridad jurídica y la gobernabilidad indispensables. Para lograr ello, es indiscutible la necesaria profesionalización de los servidores públicos que forman parte de los aparatos administrativos. En este contexto, cobra vida una figura como lo es el servicio civil de carrera, sobre todo en países en los que todavía no existe, la cual regulariza mecanismos de ingreso y selecciona a las personas por sus méritos versus patronazgo, cuotas partidistas 0 sistema de botín. 


\section{Análisis de la situación actual para el establecimiento de un servicio civil de carrera (diagnóstico)}

La Administración Pública mexicana, no obstante haber pasado un largo período de reforma administrativa (1970-1982) y de modernización (1984-2000), y haber logrado una serie de avances tanto en aspectos legales, organizacionales, procedimentales, como tecnológicos, en aras de contar con aparatos administrativos eficientes en sus tres niveles de gobierno: federal, estatal, municipal, todavía tiene asignaturas pendientes. La más importante, sin duda alguna, es la de crear un servicio civil de carrera. La ausencia de este tipo de figuras y de otras propias del modelo burocrático weberiano no sólo en M éxico sino en Latinoamérica en general, ha dado lugar, a que al gunos especialistas califiquen al proceso de reforma administrativa como de un fracaso (PRATS, 1999). Según Prats (1999), en América Latina, por lo general, no llegó a institucionalizarse sino parcial y excepcionalmente el sistema que W eBER Ilamó de dominación racional-legal encarnado en la burocracia. De ésta se tomaron las apariencias formales; pero en ausencia de los condicionamientos socio-económico-políticos necesarios, lo que se desarrollaron mayormente fueron «buropatologías», que en el mejor de los casos se aproximaban al sistema mixto que W EBER Illamó «burocracias patrimoniales».

El caso mexicano no ha sido ajeno al tipo de burocracia patrimonial de la cual nos habla PRATS - aludiendo a WEBER - , y un aspecto notorio en ese sentido es lo que acontece con la mayoría del personal en cargos preferentemente medios y altos que compone a la Administración Pública en sus tres niveles de gobierno federal, estatal y municipal y que son reclutados a través de la figura de la designación o confianza política. Fenómeno que además de darse de manera natural en períodos de elección también se presenta en etapas en las que se encuentra funcionado el gobierno electo. Los casos de cambios de secretarios de Estado en períodos intermedios o gobernadores con licencia de los Estados de la República, se constituyen en verdaderas revoluciones en las estructuras de personal de la Administración Pública.

Esta situación anacrónica está vigente - contraria a los avances logrados en otras áreas y procesos de la Administración - y sigue resultado una incógnita el que no se haya configurado el establecimiento de un servicio civil de carrera y en consecuencia, se siga al argando todavía más la distancia que se guarda con Administraciones Públicas de países avanzados, a tal grado que a la Administración Pública mexicana en este rubro se le identifique por las características que presenta, como una Administración
Pública del siglo XIX (Barzelay, 1998). En virtud de lo anterior, la pregunta obligada es: ¿cuáles son las razones por las que no se ha podido establecer un servicio civil de carrera en el país? Para ello, se identifican básicamente tres razones: razones políticas, razones jurídicas y razones administrativas.

\section{Razones políticas}

Con respecto a las razones políticas se distinguen dos problemas: uno, de carácter funcional, quetiene que ver con la falta de autonomía de la Administración Pública y el otro con la forma en que se reclutan a los servidores públicos en M éxico. En cuanto al primer problema, se puede decir que en gran parte se debió al ejercicio gubernamental-unipartidista que prevaleció por mucho tiempo en el país. Un ejercicio que en aras del proyecto nacional revolucionario dispuso de todo, sin ningún tipo de cuestionamiento, y en el que el corporativismo sindical y el de las organizaciones sociales fueron fundamentales. Por ejemplo, el papel de la Federación de Sindicatos de Trabajadores al Servicio del Estado (FST SE)5 y del Partido Revolucionario Institucional jugaron un papel esencial como órganos de legitimación política de toda acción de gobierno. Esta situación determinaba que en la realidad no había una clara distinción entre política partidista y Administración, ya que en ambas los intereses eran los mismos; en el que además el sistema de patronazgo o botín eran preponderantes iEn esa situación era necesario un servicio civil de carrera? Se considera que no la había.

Al respecto, Juan Pablo GuerRero Am parán (1999: 14-15) señala que, por lo menos, desde el sexenio de Miguel Alemán (1946-1952), en M éxico no ha estado clara la distinción entre los políticos electos y los altos funcionarios públicos, dado que la carrera política que ha llevado a la presidencia ha sido preponderantemente burocrática. Lo anterior se debe a que el sistema político era autoritario y presidencialista, con la subordinación del poder legislativo y con lógicas de competencia por el poder particulares y alejadas, hasta hace muy poco (1988), del juego de mocrático electoral.

En este marco, la Administración Pública tuvo por lo menos cinco roles esenciales para el sistema político (G UerRero Am PARÁN, 1999: 16-18):

1. La Administración Pública se convirtió en el escenario de la contienda política, particularmente la sucesión presidencial, en el que los secretarios de Estado eran los políticos contendientes.

2. Fue el sitio de la representación corporativista y clientelar, y sustituyó al congreso en ese papel de representación.

3. Además de ser enlace entre el poder político y los sectores sociales, fue también sitio de la negociación para la asig- 
nación de esos recursos. La asignación de los recursos no se negociaba tampoco ni con los representantes distritales ni a nivel territorial. Todo ello ocurría en las secretarías de Estado y en sus aparatos administrativos. D esde la perspectiva de los secretarios de Estado, la fidelidad de los burócratas era fundamental para atender a las clientelas, los grupos de apoyo político y favorecer los fines particulares de promoción política.

4. El esquema de incentivos para los funcionarios, los individuos y los sectores sociales (costos de oportunidad de cumplir plenamente con las reglas y la falta de aplicación de sanciones), favoreció enteramente el ajuste mutuo por la vía económica, particular y por encima de la ley (por cierto, extremadamente abigarrada). La Administración Pública fue el medio idóneo para el enriquecimiento de los sectores sociales que la han compuesto. Al mismo tiempo, la burocracia sale internamente cohesionada e infranqueable a consecuencia de su colusión. Es decir que esto provee de un poderoso factor de cohesión interna dentro de la burocracia y frente al exterior, por las mutuas complicidades; a su vez, resta autonomía a los burócratas en el interior del sistema y frente a los superiores.

5. Finalmente, la Administración Pública fue el brazo ejecutor incondicional del presidente. Esta moneda tiene dos caras, por el lado virtuoso, se generó un control estricto y vertical de los secretarios (y del presidente) sobre los administradores de los programas y políticas en los casos que interesaban particularmenteal jefe jerárquico. En este caso, se redujeron sustancialmente los riesgos de que la aplicación no reflejara fielmente los objetivos buscados por los mandos superiores (que no forzosamente son los objetivos más deseables para los afectados por la política). Pero existe la otra cara de la moneda. Cuando los jefes no estaban personalmente - 0 con sus servicios más allegados- supervisando la ejecución de la política, resultaba muy difícil controlar a las Administraciones. Entonces estas últimas se veían fácilmente perme adas - capturadas - por los intereses particulares de los sectores sociales interesados o por los mismos burócratas menores, una fácil presa de la corrupción (es el caso de la policía, de los órganos encargados de los permisos, las supervisiones, la administración de justicia, etc.).

Cada uno de estos roles fueron en contra de la autonomización de la Administración Pública y por ende de la posibilidad de instaurar un servicio civil de carrera, que en cierta medida hubiera separado a la Administración de la competencia política y el cual también hubiera aislado y ofrecido neutralidad a los cuerpos burocráticos y condiciones e incentivos para el ejercicio honesto y apegado a la legalidad que exige la Administración Pública.

D e los roles esenciales señalados y sobre todo el punto de la autonomización, se considera que radica la clave para entender la necesidad de que primero se requería democratizar al sistema político y posteriormente la Administración Pública incluso a través de un servicio civil de carrera. N egar la dependencia de la Administración Pública del sistema político - sobre todo de los partidos- y otorgarle una autonomía que nunca había tenido - por ejemplo a través de las reformas administrativas-, era simplemente tratar de crear una ilusión óptica.

Con respecto al segundo problema que tiene que ver con la forma en que se reclutan a los servidores públicos en M éxico, el ingreso al servicio público se realiza por conocimiento de las personas, por cuotas partidistas, compromisos políticos entre los servidores públicos, o por recomendaciones. Esto ha dado lugar, a lo quedenomina M auricio M ERIno (1996: 8-9) como la subcultura administrativa de la lealtad individual; una dinámica que fue ciertamente funcional durante una larga época de la Administración Pública mexicana y que se sustentaba en el privilegio de valores como la lealtad y la disciplina de grupo, puestos por encima de la especial idad requerida para el desempeño de una función 0 nivel de profesionalismo de quien tenía un cargo específico.

Aunque esta situación ha venido cambiado en los últimos años, por el nivel de especialización de al gunas áreas y de sus servidores públicos, se considera que, hasta hoy día, sigue vigente la idea en muchos servidores públicos del sentido de pertenencia a un proyecto político compartido, y la identidad personal del colaborador con el líder formal de la organización pública como dos elementos sine qua non para el desarrollo profesional. Éste es todavía un componente muy fuerte de la cultura política-administrativa de las personas.

De igual forma, la subcultura de la lealtad tiene también otros impactos, por ejemplo, en el sentido de competencia y cooperación que se da entre las distintas dependencias y en sus diferentes áreas del sector público. Competencia que más de las veces no es leal. D e acuerdo con ese mecanismo, cada uno de los programas implementados por las oficinas gubernamentales constituye de hecho una herramienta para la construcción de poder político y para competir en la práctica con otras oficinas públicas que en el fondo persiguen el mismo objetivo. Prácticamente, lo que se crean son nichos de poder al interior de las áreas de las Administraciones en los tres niveles de gobierno. De ahí que las redes de cooperación entre distintas instancias gubernamentales no se definan tanto por las afinidades intrínsecas a los objetivos sociales que cada una persigue, cuanto por los lazos de amistad y por las alianzas políticas establecidas entre los jefes de cada equipo administrativo.

La idea, en todo caso, de implementar un servicio civil de carrera es que tendría como finalidad modificar actitudes, comportamientos, niveles de responsabilidad, lealtades, crear una nueva cultura del servidor público pero con la condición de que ésta también haya sido modificada desde su seno: el sistema político a través del factor democratizador. 


\section{Razones jurídicas}

Con respecto a las razones jurídicas, las cuales guardan una relación directa con la legislación laboral burocrática prevaleciente, su enorme confusión por la existencia de múltiples ordenamientos jurídicos y marcada diferenciación sobre la situación de los servidores públicos de acuerdo con lo que señala el artículo 123 constitucional y la Ley Federal deTrabajadores al Servicio del Estado, han sido también razones claves para que no se haya instituido el servicio civil de carrera.

Efectivamente, la existencia de una legislación dispersa, que constituye lo que Guillermo H ARO BÉLCHEZ (1991: 19) Ilama un «aberinto jurídico «ue se materializa en falta de uniformidad, ha sido un factor preponderante para la falta de reglas claras que regulen la vida laboral de los servidores públicos. Por mencionar uno de los tantos ejemplos: el artículo 123 constitucional se divide en dos grandes apartados, el apartado A que regula las situación laboral de todo trabajador y el apartado $\mathrm{B}$ que regula la situación laboral de los trabajadores al servicio del Estado. Sin embargo, la confusión comienza cuando en el apartado A que si bien indica el propio texto constitucional: «El Congreso de la Unión, sin contravenir a las bases siguientes, deberá expedir leves sobre el trabajo, las cuales regirán: A. Entre los obreros, jornale ros, empleados, domésticos, artesanos y, de una manera general, todo contrato de trabajo», curiosamente también regula la relación laboral de los empleados públicos de la Administración Pública descentralizada, salvo los que prestan servicios públicos ${ }^{6}$. Cabe decir, que en cuanto a derechos colectivos, los trabaiadores del apartado A cuentan con una mayor amplitud para la sindicación, la contratación colectiva y el derecho de huelga, así como un régimen específico de seguridad social a cargo del Instituto M exicano del Seguro Social IM SS (BÉLCHEZ, 2000: 142). Por lo que a los burócratas que son regulados a través de este apartado se les ha llegado a denominar como burócratas de «primer piso» a diferencia de los que son regulados por el apartado $B$.

Con respecto al apartado B del artículo 123 constitucional, que establece los derechos de los trabajadores de los poderes de la Unión y el Gobierno del Distrito Federal, así como el banco central y las entidades de la Administración Pública Federal del sistema bancario mexicano que prestan su servicio al Estado, comprende a los servidores públicos de base (o sindicalizados) de la Administración Pública centralizada, mas no contempla al personal de confianza que se conforma de todos los mandos me dios y superiores; particularmente esto se corrobora en la ley re glamentaria de este apartado que lleva por título Ley Federal de Trabajadores al Servicio del Estado que establece en algunos de los artículos lo siguiente:

En su artículo 40 establece dos grupos de trabajadores: los de confianza y los de base. En su artículo 50 define como trabajadores de confianza a aquellos cuyo nombramiento o ejercicio re- quiere la aprobación expresa del Presidente de la República y los tipifica como a los que se confiere poder de decisión en el ejercicio del mando a nivel de Director G eneral, Directores de Area, Adjunto, Subdirectores y Jefes de D epartamento. Incluye también los puestos que desempeñan las funciones de Inspección, Vigilancia, Fiscalización, M anejo de Valores, Auditoría, Control de Adquisiciones; Almacenes e Inventarios; Investigación Científica; A sesoría de M andos Superiores; Secretarías Particulares; Agentes del M inisterio Público; las Policías y los miembros del Poder Legislativo y Judicial. Por su parte, el artículo 60 establece, que los trabajadores de base son todos los no incluidos en el artículo 0 - y serán inamovibles.

Por último, en cuanto a este ordenamiento, de manera expresa en el artículo 8 se establece «Q uedan excluidos del régimen de esta ley: los trabajadores de confianza, los miembros del Ejército y Armada...». Por lo cual no están establecidos cuáles son los derechos y el procedimiento a que debe sujetarse su baja o cese en el desempeño del servicio, por lo que puede considerarse que la relación del personal de confianza con la dependencia o institución es de carácter eminentemente administrativo y el cese es sólo un acto de autoridad; como consecuencia los conflictos entre los empleados de confianza y la dependencia para la cual prestan sus servicios, sobre la estabilidad de sus empleo no pueden ser resueltos por los tribunales laborales sino por jueces federales; así, antes de ocurrir al amparo o habeas corpus, deben hacerlo ante el Tribunal Federal de Conciliación y Arbitraje ${ }^{7}$, sin embargo, esto no está determinado por la Constitución.

Adicionalmente cabe señalar que la H onorable Suprema Corte de Justicia de la N ación precisa que los «trabajadores al servicio del Estado de confianza no están protegidos por el apartado B del artículo 123 en cuanto a la estabilidad en el empleo» ${ }^{8}$. El artículo 124 de la Ley Federal de Trabajadores al Servicio del Estado establece que el Tribunal Federal de Conciliación y Arbitraje (TFCA) es competente para conocer los conflictos individuales que se susciten entre titulares de una dependencia y sus trabajadores - se refiere a los de base- por la exclusión que se hace de los de confianza en el artículo $8^{\circ}$. En consecuencia el TFCA no incurre en violación de garantías en los casos de sobreseimiento del pago de indemnización constitucional y salarios caídos reclamados por un trabaiador de confianza, con un despido injustificado (BÉLCHEz, 2000: 144-145).

A pesar de estas circunstancias, los trabajadores de confianza cuya función o nivel se encuentre ubicado en el rango de los que describe la Ley, al menos cuentan con las medidas de protección al salario y los beneficios de seguridad social. Sobre el rubro de prestaciones socioeconómicas se otorgan con base en la Ley del Instituto de Seguridad y Servicios Sociales de los Trabajadores del Estado y se hacen extensivas a los trabajadores al servicio civil de las dependencias y de las entidades de la Administración Pública Federal que por ley o por acuerdo del Ejecutivo Federal se 
incorporen a su régimen, así como a los pensionistas y a los familiares derechohabientes de unos y otros. La misma ley precisa como trabajador a toda persona que preste sus servicios en las dependencias 0 entidades, mediante designación legal o nombramiento o por estar incluido en las listas de raya de los trabajadores temporales, con excepción de aquellos que presten sus servicios mediante contrato sujeto a la legislación común y a los que perciban emolumentos exclusivamente a cargo a la partida de honorarios, los cuales serán sujetos de la ley, además de los pensionistas y derechohabientes, para lo cual se entiende la inclusión tanto de trabajadores de base como de confianza.

Por otra parte, otro elemento que forma parte de la proble mática existente es que hay un régimen particular para los diferentes tipos de servidores públicos, hay uno para las fuerzas armadas, otro para los empleados bancarios, obviamente de los bancos que todavía son del Estado, para los miembros del Servicio Exterior, marina nacional, etc. Tal existencia de normas es propiciada por la propia normatividad en la materia, la fracción XIII del apartado B del artículo 123 constitucional establece que ciertas dependencias de la Administración Pública Federal se regirán por sus propias leyes, tal es el caso de la secretarías de Relaciones Exteriores, de la D efensa Nacional y Marina aś como el M inisterio Público y la Policía Judicial; la fracción XIII-Bis señala lo mismo, en cuanto instituciones que prestan el servicio de banca y crédito, Banco de M éxico y Patronato del Ahorro $\mathrm{N}$ acional. Esta situación se repite para el caso de órganos desconcentrados como el Instituto N acional de Estadística, G eografía e Informática (IN EGI) u órganos autónomos como el Instituto Federal Electoral. En palabras de Guillermo H ARo BÉLCHEZ (1991: 19) se concluye: «El laberinto termina por convertirse en un auténtico callejón sin salida al convivir con esta diversidad legislativa toda una gama de reglamentaciones propias de cada una de las dependencias de la Administración Pública Centralizada».

Para dar una idea de la existencia de diversos ordenamientos jurídicos para regular la función pública, a continuación se enuncian al gunos de los más significativos:

- Constitución Política de los Estados U nidos M exicanos, artículo 123 constitucional. Diario 0 ficial de la Federación. Diario 0 ficial de la Federación (D.O.F.) 5 de febrero de 1917.

- Ley Federal de los Trabajadores al Servicio del Estado, Re glamentaria del Apartado «B» del artículo 123 constitucional, D.0.F. 28 de diciembre de 1963.

- Ley Federal del Trabajo Reglamentaria del artículo 123 apartado A. D.0 .F. 10 de abril de 1970.

- Ley Reglamentaria de la fracción XIII Bis del apartado B del artículo 123 de la Constitución Política de los Estados
Unidos M exicanos (trabajadores bancarios) D.0.F. 30 de diciembre de 1983.

- Ley del Instituto de Seguridad Social para las Fuerzas Armadas. D.0.F. 28 de mayo de 1976.

- Ley para la Comprobación, Ajuste y Cómputo de Servicios en el Ejército y Fuerza Área M exicanos. D .0 .F. 12 de diciembre de 1942.

- Ley para la Comprobación, Ajuste y Cómputo de Servicios de la Armada de M éxico. D .0 F. 24 de diciembre de 1984.

- Ley de Ascensos de la Armada de M éxico. D .0 F.F. 14 de enero de 1985.

- Ley de Ascensos y Recompensas del Ejército y Fuerza Área M exicana. D.0.F. 7 de enero de 1956.

- Ley de Recompensas de la Armada de M éxico. D .0 F. 14 de enero de 1985.

- Ley de Premios, Estímulos y Recompensas Civiles. D.0.F. 31 de diciembre de 1975.

- Ley del Servicio Exterior M exicano. D.O F. 4 de enero de 1994.

- Ley Federal de Responsabilidades de los Servidores Públicos. D.O.F. 31 de diciembre de 1982.

- Reglamento de Vacaciones del Ejército y Fuerza Área M exicanos. D. O.F. 2 de abril de 1984.

- Reglamento de Estímulos Sociales y Económicos del M inisterio Público Federal. D.0.F. 17 de mayo de 1993.

- Reglamento de los Centros Federales de Readaptación Social. D.0.F. 30 de agosto de 1991.

- Reglamento de Reclusorios y Centros de Readaptación Social del D istrito Federal. D.0 F. 20 de febrero de 1990.

- Reglamento de Estímulos Sociales y Económico de la Policía)udicial Federal. D.0.F. 26 de marzo de 1993.

Además de las Leyes 0 rgánicas, Estatutos y Reglas que regulan a los organismos autónomos, descentralizados y desconcentrados en materia de administración de recursos humanos. Aunado a estos ordenamientos habría que mencionar a los de los Estados de la República, para lo cual la Constitución Política de los Estados U nidos M exicanos establece en su artículo 71, fracción III, que es competencia de los Estados iniciar leyes y decretos. A su vez, el artículo 73, fracción X, fija como facultad del Congreso la expedición de leyes de trabajo reglamentarias del artículo 123, y el párrafo segundo de fracción VIII del artículo 115 determina que las relaciones de trabajo entre municipios y sus trabajadores se regirán por las leyes que expidan las legislaturas locales, con base en lo dispuesto en el mencionado artículo 
123 de la Constitución y sus disposiciones reglamentarias; y con mayor precisión el artículo 16 constitucional en su fracción VI indica que «las relaciones de trabajo entre Estados y sus trabajadores se regirán por las leyes que expidan las legislaturas, los Estados con base en lo dispuesto por el artículo 123 de la Constitución Política de los Estados Unidos Mexicanos y sus disposiciones reglamentarias,...». D erivado de lo anterior, los congresos de los Estados han expedido diversos ordenamientos en los que se regulan las relaciones laborales con sus servidores públicos. Los cuales desafortunadamente, en su mayoría están hechos con criterios muy particulares y dispares, por lo que existe la situación de que no se apegan a los principios que dicta el apartado B del art. 123 constitucional (BÉLCHEZ, 1991: 20).

Cabe destacar que ocho Estados denominan al ordenamiento como Ley del Servicio Civil: Baja C alifornia, Chiapas, M orelos, Nuevo León, 0 axaca, Sonora, Veracruz y Zacatecas. Sin embargo, lo que pudiera permitir un servicio civil de carrera como tal, 0 la existencia de una carrera administrativa, aún no es contemplada en una regulación integral para todos los servidores públicos delaAdministración Pública estatal (BÉlCH Ez, 2000: 162).

Finalmente, en cuanto al derecho a la sindicación de los trabajadores al servicio del Estado tiene como base constitucional lo se ñalado en la fracción X del artículo 123 propio apartado B, el cual a la letra indica: «Los trabajadores tendrán derecho de asociarse para la defensa de sus intereses comunes. Podrán, asimismo, hacer uso del derecho dehuelga, previo al cumplimiento de los requisitos que determine la ley, respecto de una o varias dependencias de los poderes públicos, cuando se violen de manera general y sistemática los derechos que este artículo les consagrar. El Estado reconoce a la sindicalización como un derecho. Sin embargo, la normatividad y organización sindical a la que hace referencia este apartado es sólo del personal reconocido como de base, ya que los trabajadores de confianza, como lo estableceel artículo 70 de la LFFSE, no pueden formar parte de ningún sindicato y, en el caso de que algún trabajador de base pasara a ocupar un puesto de confianza y gozara de la sindicalización, para éste quedarán en suspenso todas sus obligaciones y derechos convenidos con su organización sindical. En este aspecto, tampoco se descarta la posibilidad de legislar sobre el dere cho a la sindicación de los trabajadores de confianza.

En virtud de todo lo anterior, son urgentes las reformas al artículo 123 constitucional apartado B, con el propósito de que se genere una nueva legislación con las siguientes características: que sea incluyente (por ejemplo, que no deje fuera a los servidores públicos de confianza), uniforme (que contenga criterios y lineamientos únicos y homogéneos) y general (que no haga distinciones de servidores públicos). Pero, sobre todo, que dé lugar propiamente a un servicio civil de carrera: como la implementación de nuevos mecanismos de selección e ingreso, estabilidad en el empleo, nuevos derechos y obligaciones, desarrollo profesional, carrera administrativa, premios e incentivos y retiro digno.

\section{Razones administrativas}

Sobre las razones administrativas, habría que decir que están ligadas a la falta de una cultura administrativa basada en la eficiencia, eficacia, calidad en el servicio, y apertura a la opinión ciudadana. Por el contrario, la Administración Pública ha adquirido una imagen de desprestigio creada por la corrupción, la ineficiencia, el autoritarismo, el nepotismo y el patrimonialismo. Términos contrarios al espíritu de un servicio civil de carrera.

D e igual forma, los medios por lo que se accede a la Administración Pública y la posición política de una gran parte de los servidores públicos, también genera disfunciones de tipo administrativo. Las más conocidas están asociadas a la improvisación con la que se desempeñan no pocos funcionarios de todos los niveles, con el costoso aprendizaje que se transforma en acciones ineficaces y malos resultados para la ciudadanía. Por otra parte, también está el mecanismo de evaluación sobreel desempeño de los servidores públicos, que las más de las veces es aplicado por los jefes inmediatos, dejando de lado procedimientos claros y objetivos que efectivamente demuestren la capacidad o incapacidad de los servidores públicos.

Por otra parte, de acuerdo con José Juan Sánchez (1999: 232): «el sistema de remuneraciones para los servidores públicos no es acorde con los sueldos del mercado, de tal manera que el ingreso de las personas que toman decisiones de carácter nacional no se refleja en su ingreso, que son muy similares a los que tiene una persona con el nivel bajo de una gerencia, en una empresa mediana o pequeña de la iniciativa privada. Se tienen salarios poco competitivos. La descapitalización del recurso humano dentro del sector público cada día atrae a gente con menos aptitudes o calificaciones y, algo muy importante, se generan o provocan conductas ilícitas dentro del sector, o prácticas de corrupción. Sobre este punto, de acuerdo con el indice de Percepción de Corrupción que preparó Transparencia Internacional para el año 2000, M éxico se ubica en un sitio nada favorable, con una calificación de 3.3. en una escala de 10. Esto lo ubica en el lugar 59 de un total de 90 países considerados en la investigación que realiza dicho organismo9. En el año 2001, la misma institución ubicó a M éxico en lugar número 51 entre 90 países, con una calificación de 3.7 dentro del mismo índice, ocho lugares arriba con respecto al año anterior ${ }^{10}$.

De acuerdo con lo que señala el Programa Nacional de Combate a la Corrupción y Fomento a la Transparencia y el Desarrollo Administrativo 2001-2006 de la Secretaría de la Contraloría y D esarrollo Administrativo, si bien es difícil cuantificar el tamaño del problema de la corrupción en M éxico, ya que no se han hecho estudios al respecto, si existen algunos indicadores que son marco de referencia para dimensionarlo. En efecto, el Banco M undial estima que representa el $9 \%$ del PIB nacional, además de haber una fundada percepción en la opinión 
pública de que existe corrupción y que ésta genera ineficiencias en la actividad pública y servicios de baja calidad; por lo que se trata de un fenómeno de magnitud importante.

0 tras de las prácticas que existen en el personal que se desarrolla en la Administración Pública y que no percibe sueldos elevados, es que cuenten con dos o más fuentes de ingreso adicionales, a veces dentro de la propia Administración Pública, lo que trae como consecuencia que los puestos que se ocupan no desarrollen de manera óptima la funciones que debiesen cumplir, y no haya un verdadero compromiso de los servidores públicos con la institución.

También se carece de un sistema de desarrollo del servidor público dentro de las organizaciones. Esto significa que no existe un sistema que califique y reconozca el desarrollo del servidor público, sobre todo si éste ha adquirido mayores conocimientos o habilidades dentro de la función pública que desempeña; que le permita ir ascendiendo a niveles superiores. La ausencia de instrumentos objetivos de evaluación del desempeño, también cancela la posibilidad de promover a las personas a puestos supe riores. El sistema no está basado en el mérito sino en la lealtad personal.

Todo lo anterior se puede corroborar y sintetizar en el diagnóstico sobre la problemática actual de la Administración Pública Federal realizado por la Secretaría de H acienda. Al respecto, la Secretaría señala que los principales puntos problemáticos se dividen en cuatro esferas (GuerRERo Am PARÁN, 1997: 21):

1. Las compensaciones son el primer motivo de preocupación. El sueldo que perciben los funcionarios públicos se divide entre el sueldo base muy poco remunerado y que cuenta con fundamento legal, y las compensaciones, que constituyen la mayor proporción del ingreso, pero no tie nen un respaldo legal sólido. La uniformidad en el sueldo según el nivel jerárquico no contempla las diferencias en el grado de responsabilidad. Y el monto de los salarios insuficiente frente al mercado de trabajo constituye una de las fuentes de corrupción, en el diagnóstico de Hacienda.

2. La contratación de los funcionarios públicos no obedece a un esquema único que regule los procesos de selección, reclutamiento, ingreso, desarrollo profesional y separación. Prevalece la discrecionalidad del superior jerárquico que contrata y controla verticalmente a sus subalternos, que generalmente sólo responden ante él.

3. El desarrollo profesional de los servidores públicos no cuenta con una visión estratégica de largo plazo. La carre ra depende del jefe administrativo del grupo del cual forma parte el funcionamiento. Si el superior es removido 0 reubicado, igual suerte corren sus subalternos.

4. La separación es conflictiva, pues no hay ningún tipo de seguridad en el empleo para los funcionarios de confianza. Además constituye otra fuente de corrupción, pues la inseguridad en el empleo es un incentivo para usufructuar, aunque sea al margen de las normas, los recursos públicos y la información privilegiada de esos puestos.

0 tro problema de carácter administrativo es el que se registra con personal que goza de inamovilidad en el puesto (puestos de apoyo administrativo, secretarias, auxiliares, mensajeros, en al guno casos analistas, jefe de oficina, etc.), que la mayoría de las veces es manejado por personal sindicalizado. Si bien no se trata de subvalorar al personal sindicalizado, por la posición que goza la mayoría de este personal, la realidad indica que más de las veces no ejercen adecuadamente las actividades que se le encomiendan. Este problema es muy palpable en instituciones que cuentan con un gran número de personal sindicalizado. Un ejemplo de ello lo es el Gobierno del Distrito Federal que cuenta entre sus filas con 109.000 mil trabajadores sindicalizados de una estructura total de 283.980 mil servidores públicos (datos de la Dirección General de Administración y D esarrollo de Personal del Gobierno del D istrito Federal), que representa poco menos de la mitad de la estructura del personal. Sin embargo, el problema se agudiza aún más por el peso que tienen casi todas las secciones del Sindicato Ú nico de Trabajadores del Gobierno del Distrito Federal (SUTGDF), que prácticamente en más de una vez han cerrado instalaciones completas del Gobierno del Distrito Federal por demandas no satisfechas; situación como la antes descrita ha paralizado las actividades y por ende ha generado perjuicios a la ciudadanía, sobre todo si de por medio existen trámites por resolver. De igual forma, el fantasma del corporativismo también ha hecho acto de presencia, sobre todo cuando un sindicato como el SUTGDF con tintes priístas (tal como los señalan sus Estatutos), simplemente no coincide en proyectos del gobierno actual del Distrito Federal, que es un gobierno de signo partidista distinto, por lo que las dificultades de coordinar funciones y para gobernar la Ciudad de M éxico son mayores.

Por último, está la inexistencia de metas y cooperación intersecretariales 0 interorganizacionales. En la práctica, las oficinas públicas no suelen establecer vínculos formales ni procedimientos de cooperación asentados en la cercanía de sus propósitos públicos, a menos que haya razones políticas específicas. Por el contrario, la práctica más frecuente es que cada dependencia formule sus propios programas y trate de implementarlos sobre la base de sus propios recursos, aun cuando sea evidente que podría al canzar mejores resultados mediante la cooperación con alguna otra dependencia complementaria. Al mismo tiempo, esa preferencia por la exclusividad cuando por el aislamiento tiene su correlato en el trato generalmente discreto - cuando no secreto- de la información que se produce en cada una de las dependencias. D e tal forma, que la competencia por los espacios políticos suele imponerse sobre las posibilidades de cooperación 
entre órganos de gobierno, incluso dentro de una sola dependencia (M ERIN 0, 1996: 13-14). Los ejemplos abundan, están los casos en los que al buscarse una solución a un desastre ecológico, es notoria la falta de coordinación y cooperación que da entre las tres instancias de gobierno. Todo este manejo político y administrativo poco eficiente, falta de transparencia y falta de cooperación tienen por añadidura una repercusión en el diseño e imple mentación de políticas públicas.

\section{El servicio civil de carrera en el gobierno de Vicente Fox}

Con respecto al gobierno federal actual del presidente $V$ icente Fox Q uesada, quien tomó posesión del cargo el primero de diciembre del 2000, el Plan Nacional de D esarrollo 2001-2006 en su apartado denominado «Buen Gobierno» señala lo siguiente en sus principales líneas:

«A fin de que esta administración cumpla con su responsabilidad histórica de dar respuesta a las grandes demandas y expectativas de la sociedad, requerimos acciones capaces de transformar radicalmente los esquemas tradicionales de gestión... El gobierno que necesitamos construir es uno de calidad total, que ponga en el centro del quehacer gubernamental la satisfacción de las necesidades y expectativas de la sociedad...Requerimos de un gobierno inteligente, capaz de utilizar los más avanzados sistemas administrativos y tecnológicos para evitar el dispendio de recursos y promover la eficacia de su función en todos los órdenes. D ispuesto a aprender de las experiencias de éxito registradas en otras partes del mundo para adecuarlas al contexto nacional, con la firme convicción de que siempre es posible mejorar... Requerimos de un gobierno ágil y flexible, capaz de captar oportunidades, atender los problemas y adecuarse a las circunstancias rápida y eficazmente...Frente al paradigma de un gobierno operativo, orientado a administrar normas y procesos, prevalezca el de un gobierno estratégico, orientado a administrar resultados. Requerimos que los procesos burocráticos que inhiben el desarrollo de los actores generadores de riqueza se rediseñen con un enfoque de calidad para atender las necesidades del ciudadano. La rigidez tiene que ser sustituida por flexibilidad, la cual nos permitirá derribar las barreras existentes para la innovación y creatividad, impuestas por la excesiva normatividad y sobrerregulación existentes... Requerimos de un gobierno descentralizado, que en sus relaciones con los estados y municipios propicie no sólo una simple desconcentración de funciones, sino un nuevo pacto federal, que deje en claro que la nación es más que la suma de sus partes... En suma, requerimos un gobierno de clase mundial, un gobierno innovador y de calidad total. Para lograrlo, trabajaremos en dos vertientes contenidas en el modelo de innovación y calidad que se ha adoptado. Por un lado, la innovación que permita concebir nuevas formas de hacer las cosas, y por el otro, la calidad total que permita mejorar y optimizar lo que se ha estado haciendo bien y ha demostrado que genera valor agregado a la sociedad».

De este plan básicamente se desprenden dos documentos que representan y sobre los que gira la propuesta modernizadora de la Administración Pública Federal. Uno de ellos, es el Programa N acional de Combate a la Corrupción y Fomento a la Transparencia y el Desarrollo Administrativo 2001-2006 PN CTDA cuya responsabilidad de ejecución la tiene la Secretaría de la Contral oría y D esarrollo Administrativo Secodam y el otro es el «M odelo Estratégico para la Innovación Gubernamental» diseñado por la 0 ficina de la Presidencia para la Innovación Gubernamental», la cual tiene no el rango de secretaría sino de órgano staff del Poder Ejecutivo Federal.

Sobre el primer documento que es el PN CTDA, tiene como fundamento el combate a la corrupción a partir de la utilización de un enfoque de carácter preventivo, en este tenor, se destacan los proyectos de creación de un Código de Ética para los servidores públicos, de una nueva Ley Federal de Responsabilidades Administrativas de los Servidores Públicos, de la Ley Federal de Acceso a la Información y de Transparencia en la G estión Pública, y la creación de figuras como las Contralorías Sociales con el fin de controlar y evaluar las acciones de la Administraciones Pública. Entre los proyectos más importantes se contempla el fortalecimiento de Sistemas de C ontrol Interno en la Administración Pública Federal el uso de la tecnología para la Transparencia en el Gobierno (compranet, traminet, declaranet, normativanet), la coordinación con Estados y municipios para vigilar la transparencia en la transferencia de recursos, la información pública sobre la operación y desempeño de las instituciones y los servidores públicos, la medición del impacto en el combate a la corrupción e impunidad, la incorporación de los temas de combate a la corrupción y a la impunidad y de ética pública en el Sistema Educativo Nacional, las relaciones institucionales de vinculación para la transparencia y la creación del Consejo $\mathrm{Na}$ cional de Transparencia y contra la corrupción.

Sobre el tema que nos interesa, el programa contempla la creación de una Ley del Servicio Civil de Carrera así como la implementación de la carrera administrativa. Esta medida estará acompañada de acciones de igual importancia como el estudio de sueldos y salarios de las áreas criticas de la Administración Pública Federal para evitar prácticas de corrupción, la formación y capacitación de servidores públicos y la inducción y adopción voluntaria del Código de Ética de los servidores públicos.

Con relación al segundo documento que es el «M odelo Es- 
tratégico para la Innovación Gubernamental» cuya responsabilidad de ejecución corresponde a la 0 ficina de la Presidencia para la Innovación Gubernamental, según el documento, es un modelo de innovación social para la Administración Pública para propiciar un cambio en la misma. La innovación gubernamental, según el modelo, es el movimiento cultural que busca transformar radicalmente la orientación, la capacidad y la velocidad de respuesta estratégica de la Administración Pública Federal, re visualizando todo desde múltiples perspectivas. Sus elementos claves son: 1) Eliminar áreas y personal de la Administración que se consideren innecesarios, 2) concentrar a todos los servidores públicos en un propósito (focus); 3) está orientado a generar resultados que añadan valor al gobierno y la sociedad y 4) toma en cuenta el entorno en el que participa, afecta e impacta a la Administración Pública Federal. El focus estratégico es que la innovación del gobierno debe tener como propósito superior, mejorar la capacidad de gobernar para atender los anhelos y expectativas de las sociedad mexicana y recuperar con ello la confianza en sus autoridades.

La columna vertebral de este modelo se basa en la creación de un Código de Ética para los servidores públicos como base para implementar una política de innovación y calidad y como medio para propiciar el cambio de paradigma en la Administración Pública, y el uso intensivo de técnicas como la reingenería de procesos, el benchmarking, la calidad total y la subcontratación de servicios. Por lo que a lo largo del documento, se encontrarán términos como gobierno por resultados, «administración cruzada», «conexión emocional» (los cuales por ejemplo no se explican con claridad en el documento), focus estratégico, planeación estratégica, benchmarking, host groups y todos los términos propios de «la familia ampliada de ideas» de la que habla BARzELAY (1998) como eficiencia, eficacia, visión, resultados, flexibilidad, innovación, clientes; términos propios de los modelos gerenciales. Paradójicamente a la enorme carga de técnicas y términos ge renciales, el documento considera indispensable la creación de un servicio civil de carrera para la Administración Pública. En este punto es en el que surgen dudas de cómo conciliar reingeniería de procesos, cuya idea de acuerdo con las experiencias del sector privado tiende a reducir las plantillas (M ICKLETH WAIT y W 00 LDRIGDE, 1998), con calidad total que considera que el personal es el recurso más importante, con servicio civil de carrera que privilegia la estabilidad en el empleo.

Sobre estos dos documentos se puede concluir diciendo que en ambos es notoria la preferencia en la utilización de términos y técnicas de tipo gerencial, no obstante ello, no se desatiende la posibilidad de la implementación del servicio civil de carrera, pero simplemente como una medida más. D ebe decirse que el PNCTDA es mucho más puntual y riguroso en cuanto a objetivos, líneas estratégicas y acciones para con la concreción de esta figura. Es menester mencionar que se destaca en ambos docu- mentos la presencia de un tercer elemento que es el de la «responsabilización», considerada por algunos especialistas como parte de un tercer derrotero o paradigma de la Administración Pública (Ross, 2000), en la que figuras como los códigos de ética, el control ciudadano sobre la Administración, la supervisión legislativa, la transparencia en el manejo de recursos hacen acto de presencia. Se considera que la preferencia por el tipo de términos gerenciales utilizada en ambos documentos obedece en parte al perfil empresarial con el que cuenta el propio presidente - existen antecedentes de experiencias empresariales a nivel de gobierno en su entidad natal (G uanajuato) cuando fue gobernador- y la mayoría de los funcionarios que forman parte de su gabinete y demás áreas de la Administración, así como de la gente que forma parte de su partido, el Partido Acción Nacional PAN ; partido que tradicionalmente está identificado con la clase empresarial del país. Sólo esperemos que la mezcla entre elementos blandos (N GP) con elementos duros (SCC) no sean explosivas sino por el contrario productivas.

\section{Perspectivas}

La coyuntura de reforma institucional y de consolidación democrática por la que atraviesa un país como M éxico estállena de grandes cambios, que van desde la definición del papel del Estado y de sus órganos de poder en la sociedad hasta la transformación de las prácticas de patronazgo, patrimoniales y clientelares, vigentes hasta hoy día, para sustituirlas por las de mérito, responsabilidad, legalidad, seguridad jurídica y ciudadanía, con un cariz de competitividad, eficiencia y calidad, con el propósito de abatir el déficit de legitimidad y recuperar la confianza ciudadana. Sin lugar a dudas, la profesionalización de los servidores públicos de la Administración Pública es un proceso de mucha importancia como parte de esos cambios que se necesitan lograr.

Para ello, como ya se explicó a lo largo del artículo, existen en juego dos visiones sobre la materia, la que se pronuncia por la profesionalización a través del movimiento de la Nueva G estión Pública, en particular la G estión Estratégica de Recursos H umanos y aquella que tiene que ver con el establecimiento de un servicio civil de carrera. La problemática sobre todo de conjugar ambas visiones consiste principalmente en que estas dos posiciones no son necesariamente complementarias, por el contrario, con base en sus respectivos postulados aparentemente se rechazan de manera mutua. Se plantea así un conflicto entre dos instituciones, el modelo burocrático weberiano, fuertemente asentado en las Administraciones Públicas contemporáneas a pesar de las críticas y de los intentos de cambio, y la G estión Estratégica de Recursos H umanos soportado por el movimiento de la Nueva Gestión Pública. 
Según M iquel Salvador (2000: 17), el conflicto se genera especialmente al plantear la necesidad de preservar las funciones clásicas de la Administración Pública junto con el desarrollo de nuevas actividades de corte empresarial sobre todo dirigidas a la prestación de servicios públicos. La discusión se plantea en términos de combinar los principios básicos y comunes de la función pública clásica con las propuestas desarrolladas desde un enfoque de gestión que apuntan a la necesidad de introducir una orientación estratégica en la G estión Estratégica de Recursos H umanos, unida a cierta flexibilidad y a la capacidad de adaptación, para asegurar el propio cumplimiento de la misión (variable y cambiante) de las Administraciones Públicas. Para efectos de un cambio institucional, se podrían dar incluso dos escenarios distintos: uno que significara la ruptura de un modelo con otro, en el que se hicieran valer los valores predominantes de uno de ellos, o un escenario de tipo incremental en el que a partir de un modelo se hicieran una serie de innovaciones a partir del otro.

Al respecto, se está totalmente de acuerdo con Miquel SALVAD O R en la identificación del problema y sus posibles soluciones a través de un cambio institucional que sería la sustitución de un modelo por otro o la combinación de ambos modelos de profesionalización, pero claro está, esto sólo puede darse en Administraciones Públicas que tengan la condición de haber consolidado previamente un modelo burocrático clásico y que partir de ahí se comiencen a realizar una serie de innovaciones propias de otro estilo o quehacer administrativo llámese en este caso la N ueva Gestión Pública.

Evidentemente que la situación es distinta para un caso como M éxico - seguramente también para otros casos latinoamericanos- en la que no se puede hablar de la consolidación de un modelo clásico en ausencia de un sistema de mérito 0 un servicio civil de carrera. Sobre este punto, es de gran relevancia lo que se ñala Joan PraTs (1999): «Frente a las interesantes propuestas de este neorreformismo administrativo latinoamericano envul to de propuestas de modernización pública y de técnicas de la N ueva Gestión Pública, creemos, se tiene que adoptar una posición de diálogo crítico. Las consideraciones que siguen tienden a resal tar:

a) que para el desarrollo latinoamericano, en el que está incluido M éxico, la mayor urgencia de reforma administrativa todavía es la creación de verdaderas burocracias capaces de asumir eficazmente las funciones exclusivas del Estado en un marco de seguridad jurídica;

b) que para el desempeño de las funciones exclusivas del Estado el sistema de gestión más racional - aunque sea sólo como third best- continúa siendo la administración burocrática, aunque redescubierta y reinventada;

c) que el ámbito propio de la revolución gerencial se encuentra principalmente en el ámbito de la provisión directa de bienes y servicios públicos, que hoy es el espacio público cuantitativamente mayor, pero que no se incluye en las funciones exclusivas del Estado, cualitativamente más importantes;

d) que la revolución gerencial pública no es nunca una mera traslación de las técnicas y cultura del sector privado, ya que debe resolver problemas genuinamente «públicos» como son la dificultad del monitoreo de las relaciones entre financiamiento y provisión de servicios, las dificultades en la medición y control de resultados, la problemática inherente a los cuasimercados o mercados planificados y, finalmente, los impactos de la información y participación ciudadana».

Con todo esto, lo que se pretende decir es que en una coyuntura de cambio institucional, la revalorización de la función pública burocrática y lo que es propiamente la figura del servicio civil de carrera se vuelve un requisito ineludible tanto en países que cuentan con experiencias previas como en los que por primera vez quieren incursionar en su establecimiento. Una revalorización que, por supuesto, no está exenta de cambios, rediseños, reformas, reorientaciones y adecuaciones a los tiempos actuales.

En ese sentido, para que la Administración Pública mexicana pueda participar en un cambio institucional de tipo incremental, necesariamente se tendrá que crear las bases para ello, como lo es el establecimiento de un servicio civil de carrera y una vez creadas las bases no se descartarían de ninguna manera otros instrumentos tendientes a su mayor dinamismo. $\mathrm{Ni}$ tampoco se descartarían los diversos procesos de innovación administrativa, con diferente grado de éxito que se han dado en las distintas administraciones sobre todo a nivel local, así como las distintas técnicas de gestión de recursos humanos puestas en práctica (evaluación del rendimiento, carrera administrativa, programas de calidad vinculados al cambio en la cultura organizativa). La conveniencia y posibilidad de introducir ciertos elementos propios del enfoque de la Gestión de los Recursos Humanos, sin llegar a adoptar sus postulados como una institución integral alternativa a los valores y normas, reglas, rutinas y procesos asociados a la «función pública democrática», se plantea como una opción de cambio incremental que puede contribuir a mejorar la actividad desarrollada por las Administraciones Públicas (SAlVAD OR: 2000: 20). Se considera que éstos son los tipos de escenarios que se tienen que construir próximamente para un país como M éxico.

Los cambios que se tienen que realizar para establecer un servicio civil de carrera deben apuntar a lo siguiente:

a) Reformas a la Constitución en el artículo 123 en el Apartado $B$.

b) Reformas a la Ley Federal deTrabajadores al Servicio del Estado que le brinde seguridad jurídica a los trabajadores de confianza y que acorte diferencias entre distintos tipos de servidores públicos. 
Esta medida es de suma importancia ya que, además de brindarle derechos, estabilidad, seguridad laboral y recursos de de fensa a los servidores públicos de confianza, le brindaría una protección constitucional a todos aquellos servicios civiles de tipo sectorial - además de los ya existentes- que tuviesen que crearse por exigencias técnicas.

Por otra parte, se visual iza la existencia de diferentes servicios civiles de carrera en distintas modalidades de acuerdo con los diferentes tipos de administración, por principio esto sería a través de la promulgación de ordenamientos jurídicos:

a) La promulgación de una ley del Servicio Civil de Carrera para el Gobierno Federal o para servidores públicos de la administración central. Por otra parte, la decisión de cada uno de los organismos que forman parte de la administración paraestatal, por la personalidad jurídica y autonomía que tienen, de crear su propio servicio.

b) Q ue cada Estado de la República formule su propia ley y cuente con su propio servicio civil de carrera. De igual forma que cada Congreso Local legisle en la materia para con sus municipios.

c) Que cada órgano autónomo cuente con su propio estatuto de servicio civil de carrera.

Cabe aclarar que este esquema es sumamente general y seguramente excluye a otros tipos de administración que, ahora mismo, juegan un papel igual de importante que las otras instancias. Se puede observar, por lo tanto, la necesidad de combinar macrorreformas con microrreformas. Este esquema tiene el pro- pósito de dar sólo una idea. De igual forma, el establecimiento de un servicio civil de carrera en los distintos tipos de administración no significa que desaparezcan del todo figuras como la designación política y los puestos ocupados por los sindicatos. Se prevé que los niveles de politización de las Administraciones Públicas - federal, estatal y municipal - a través de la figura de la designación política así como de su sindicalización serán objeto de amplias negociaciones entre políticos y burócratas. D e hecho, no se descarta la tendencia que parece apuntarse en la mayoría de los países occidentales que es la de un cierto equilibrio o modelo mixto, que combinaría en proporciones variables, cesantías, clientelismo, cargos de confianza políticos y funcionarios de carrera, con trasvases a la arena política de numerosos ejecutivos de la Administración Pública, lo que una vez más confirma la habitual confusión o falta de delimitación clara entre administración y política (SubIRaTs, 1991: 35). La Administración Pública no está exenta de la dinámica descrita, sin embargo, le sigue faltando una pieza a su puzzle o rompecabezas: un sistema de mérito 0 un servicio civil de carrera.

Finalmente, se parte de la idea de que un sistema de mérito 0 un servicio civil de carrera, puede ser un instrumento que modifique valores, cultura tanto burocrática como ciudadana, intereses, actuaciones, desempeño, trato, diálogo, lenguaje y modo de vida, tendiente por supuesto a mejorar la calidad de vida de los ciudadanos y a hacer más fuerte y saludable la vida del Estado y de las instituciones. De no establecerse se estará cerrando la puerta a una buena parte de la consolidación democrática que necesita un país como M éxico. De ahí la importancia de dar los pasos y sentar las bases para establecerlo.

\begin{abstract}
* Licenciado en Ciencias Políticas y Administración Pública. H a sido galardonado con el I Premio Andrés Bello 2001, sobre estudios de investigación sobre administra ciones públicas iberoamericanas, convocado por el IN AP de España.

1 Resulta imprescindible aclarar que el término por utilizar en este artículo será el de Servicio civil de carrera, de acuerdo con la tradición política-administrativa tanto académica como la que se utiliza en el servicio público en M éxico y en la mayoría de los países latinoamericanos, esto a diferencia del utilizado en España que es el de función pública. No obstante, el término se puede prestar a confusión si se toma en cuenta la existencia de dos modelos de función pública uno de carácter cerrado y otro abierto, en el que en el primero se utiliza precisamente el termino de carrera administrativa. Por la tanto, esto puede dar lugar a que se presuma que desde un inicio la investigación se pronuncia por la idea de un servicio civil de tipo cerrado para M é xico. De ninguna manera, independientemente de estas dos formas existentes - que el término en M éxico no las distingue- , la utilización de la figura del servicio civil de carrera se hace a partir de entenderla únicamente como un sistema en el que el ingreso de las personas al servicio público se hace a través de sus méritos; cuyo lugar una vez obtenido por ellas les brindará estabilidad en el empleo y una serie de dere chos y obligaciones. Todo ello, en contraposición al sistema actual en M éxico denominado como sistema de patronazgo o en su versión más negativa como sistema de botín 0 spoil system.

2 La modernización representa todo un cambio de estrategia frente a las tentativas anteriores a la reforma administrativa. A diferencia de lo que se conoce como refor-
\end{abstract}

ma (que en más de los casos es de carácter legal) (CARRILLO, 1992: 4), la modernización trata de combinar rápidas actuaciones, rentables y viables a corto plazo, orientadas a los aspectos más superficiales con el fin de obtener así apoyos; junto con los cambios duraderos, a más largo plazo, que afectan a la cultura de las organizaciones (M etcalfe y Richards, 1989: 33). Según M anuel Villoria (1997:77), el término modernización se utiliza como paraguas conceptualizador bajo el que se acogen todo un conjunto de fenómenos de adaptación. Fenómenos que parecen intentar alumbrar un nuevo paradigma de gestión pública, una nueva forma de pensar sobre la acción de gobernar y sobre cómo ha de materializarse ésta».

3 Los avances en materia de profesionalización y propiamente en el establecimiento de un servicio civil de carrera se han dado en los siguientes organismos: Instituto Nacional de Estadística, Geografía e Informática, la Secretaría de Educación Pública (carrera magisterial), Procuraduría General de la República, Procuraduría General de Justicia del D istrito Federal, Comisión Nacional del Agua, Procuraduría Agraria, Instituto Federal Electoral y la Secretaría de H acienda y Crédito Público (Sistema de Administración Tributaria). La gran excepción por antigüedad lo es la Secretaría de Relaciones Exteriores con la existencia de la carrera diplomática que data desde 1934. 4 Se recomiendan las lecturas: «La Función Pública en Francia: Problemas y Perspectivas» de N icole DE M ON TRICH ER y «Legado H istórico y Evolución Reciente de la Función Pública» de H ans U LRICH D elIEN en Revista Internacional de Ciencias Administrativas Vol. 57 núm. 3, Septiembre 1991, «La reforma del servicio civil en las democracias avanzadas: mérito con flexibilidad» de Francisco Longo M ARTínez en 
Red de G estión Pública y Trannsparencia, Banco Interameramericano de D esarrollo Washington D.C. 2001, y Administrative and Civil Service Reform. Banco Mundial Washington D.C. 2000.

5 La FST SE está integrada por cerca de 1.700 .000 burócratas, integrados en 88 sindicatos sólo en órganos de la Administración Pública Federal (BÉlCH EZ, 2000: 174). ${ }^{6}$ La Ley Federal de Trabajadores al Servicio del Estado, en su artículo $1^{\circ}$ amplía su aplicación para organismos descentralizados como el ISSSTE, Juntas Federales de M ejoras M ateriales, Instituto N acional de Vivienda, Lotería N acional, Instituto $\mathrm{Na}$ cional Indigenista, Comisión N acional Bancaria y deValores, Comisión de Tarifas de Electricidad y $G$ as, C entro M aterno Infantil M aximino Ávila Camacho, H ospital In- fantil y los organismos descentralizados similares que tengan a su cargo función de servicio públicos.

7 Véase Volumen relativo a las «Tesis ejecutorias 1917-1985», apéndice al Semanario Judicial de la Federación, Q uinta Parte, Cuarta Sala, p. 282 y 284.

8 Volumen relativo a las «Tesis ejecutorias 1917-1985», apéndice al Semanario Judicial de la Federación, Quinta Parte, Cuarta Sala, p. 286.

9 V éase Poder Ejecutivo Federal Plan N acional de D esarrollo 2001-2006 M éxico 2001. 10 Véase Secretaría de la C ontral oría y D esarrollo Administrativo Programa N acional de Combate a la corrupción y fomento a la Transparencia y el Desarrollo Administrativo 2001-2006. M éxico 2001.

\section{Bibliografía}

Am strong, M., 1999, A H andbook of H uman Resource M anagement Practice, Koogan Page. London.

Barzelay, Michel, 1998, Atravesando la burocracia, Ed. Fondo de Cultura Económica. M éxico.

Carrillo Barroso, Ernesto, 1992, «La M odernización de las Administraciones Públicas ante la integración de Europa», en Cambio Político y Gobernabilidad, Ed. Cole gio Nacional de Ciencias Políticas y Administración Pública, A. C. M éxico.

De M ontricher, Nicole, 1991, «a Función Pública en Francia: Problemas y Perspectivass, en Revista Internacional de Ciencias Administrativas, vol. 57, núm. 3, Septiembre.

Guerrero Am parán, Juan Pablo, 1999, «Trabas y oportunidades para el servicio civil de carrera en un M éxico Democratizado», en Visión Dimensional del Servicio Público Profesionalizado, Ed. Plaza y Valdés M éxico.

Guerrero Am parán, Juan Pablo, 1997, «Un estudio de caso de reforma administra tiva en M éxico: Ios dilemas de la instauración de un Servicio Civil a nivel federal», D ocumento deTrabajo, D ivisión de Administración Pública, Ed. CIDE, M éxico.

H ARO BÉLCHEZ, Guillermo, 1991, «La vigente legislación de funcionarios en M éxico y la necesidad de su reforma», La función pública en el proceso demodernización nacional, Ed. IAPEM Edo. de M éxico.

H ARO BéLCHEZ, Guillermo, 2000, Servicio Público de Carrera. Tradición y perspectivas, Ed. Instituto $\mathrm{N}$ acional de Administración Pública, M éxico.

Klingner D onald, E. y N albadian, John, 1998, Public Personal Managment Contextsand Strategies, Ed. Prentice H all U.S.A.

LONGO, Francisco, 2001, «La reforma del servicio civil en las democracias avanzadas: mérito con flexibilidad. Red de Gestión Pública y Transparencia, D ocumento deTrabajo, Banco Interamericano de D esarrollo. Washington D.C. Abril del 2001 U.S.A.

M artínez Puón, José Rafael, 2000, «a calidad en la Administración Pública M exicana, de la calidad total a las normas ISO 9000: el camino correcto o el falso camino», Revisa Prospectiva, n. 17 noviembre del 2000 Ed. Política Nueva A.C. M éxico D.F.

M ÉNDEZ, JoséLuis, 1998, «La modernización administrativa», Revista Enlace, mayojunio 1998. M éxico.

M ÉNDEZ, José Luis, 1998, «¿Vieja o N ueva Administración Pública? Una propuesta ante los dilemas de la M odernización Administrativa», D ocumentos 0183 III Congre so Internacional del Centro Latinoamericano de Administración para el D esarrollo sobre la Reforma del Estado y de la Administración Pública, M adrid, España 14-17 de octubre de 1998, Ed. CLAD Venezuela.

M ICKLETh WAit, John y Wo L LD RIDGE, Adrian, 1998, La hora de los gurús. Visionarios y nuevos profetas de la gestión empresarial, Ed. Alianza Editorial. M adrid, España.
M eRIN O H UeRTA, M auricio, 1996, «D e la Lealtad Individual a la Responsabilidad». Ensayos sobre la Nueva Administración Pública, Revista de Administración Pública, n. 91 M éxico.

M etcalfe, Les y Richards, Sue, 1989, La M odernización dela Gestión Pública, Ed. IN AP España.

PARd 0, M aría del Carmen, 1995, «El Servicio civil de carrera en M éxico: un imperativo de la modernización». Revista Gestión y Política Pública, vol. IV, núm. 2, M éxico, segundo semestre 1995, Ed. CIDE, M éxico.

Prats, Joan, 1998, «D el Clientelismo al M érito en el Empleo Público: Análisis de un Cambio Institucional». D ocumento electrónico incluído en la Biblioteca del IIG -PNU D (http://www.iigov.org/i igov/pnud/bibliote/pbiblio.htm). Colección Textos, n-. 058.

Prats, Joan, 1999, «Reinventar la Burocracia y Construir una nueva Gerencia Pública». Documento electrónico incluido en la Biblioteca del IIG-PNUD (http://www.iligov.org/i igov/pnud/bibliote/pbiblio.htm). Colección Textos, nํ.. 063.

Ross, Ben, 2001, «La política de la reforma administrativa: dilemas insolubles y soluciones improbables». Revista del CLAD Reforma y D emocracia, n. ${ }^{\circ} 20$, junio, Ed. CLAD, Caracas Venezuela.

SALVAD OR, M iquel, 2000, «Procesos de modernización y prestación de servicios públicos. El papel de las instituciones en la gestión de las Administraciones Públicas», V Congreso Internacional del CLAD sobre la Reforma del Estado y de la Administración Pública, República D ominicana.

Sánchez González, José Juan, 1999, «Profesionalización del servicio público: antecedentes y perspectivas en M éxico». Enfoques de Políticas Públicas y Gobernabilidad, Ed. CN CP y S, ITAM y Universidad Anáhuac, M éxico.

SUBIRATS, Joan, 1991, «La Administración pública como problema. El análisis de políticas públicas como propuesta», en D ocumentación Administrativa 224-225 Políticas Públicas y organización Adminisrativa, octubre de 1990-marzo de 1991, Ed. Instituto Nacional de Administración Pública, Madrid, España.

ULRICH DELIEN, H ans, 1991, «Legado H istórico y Evolución Recientedela Función Pública», en Revista Internacional de Ciencias Administrativas, vol. 57, núm. 3, Septiembre.

Uvalle Berrones, Ricardo, 2000, Institucionalidad y profesionalización del servicio público en M éxico. Ed. Plaza y Valdés M éxico.

VILLORIA, M anuel, 1997, «M odernización Administrativa y Gobierno Potsburocrático» en la Nueva Administración Pública (Rafael Bañon y Ernesto Carrillo, comps.), Ed. Alianza Universidad Textos, M adrid España.

W ORLD BANK, 2000, Administrative and Civil Service Reform. Washington D.C. USA. 
